\title{
Drift by dichotomous Markov noise
}

\author{
I. Bena \\ Département de Physique Théorique, Université de Genève, CH-1211 Genève 4, Switzerland \\ C. Van den Broeck \\ Limburgs Universitair Centrum, B-3590 Diepenbeek, Belgium \\ R. Kawai \\ Department of Physics, University of Alabama at Birmingham, Birmingham, Alabama 35294, USA
}

Katja Lindenberg

Department of Chemistry and Biochemistry 0340 and Institute for Nonlinear Science, University of California, San Diego, La Jolla, California 92093, USA

(Received 26 May 2003; published 28 October 2003)

\begin{abstract}
We derive explicit results for the asymptotic probability density and drift velocity in systems driven by dichotomous Markov noise, including the situation in which the asymptotic dynamics crosses unstable fixed points. The results are illustrated on the problem of the rocking ratchet.
\end{abstract}

DOI: 10.1103/PhysRevE.68.041111

PACS number(s): 05.40.-a, 02.50.-r

\section{INTRODUCTION}

Brownian motion is one of the paradigms of statistical mechanics. Following the seminal works of Einstein, Langevin, and Smoluchowsky, a detailed mathematical analysis can be made on the basis of Langevin or Fokker-Planck equations. While Brownian motion and its "time derivative," Gaussian white noise, are stochastic processes of fundamental importance, the dichotomous Markov process (see, e.g., Ref. [1]) has its own virtues and interest. First-and this is our central contention here-systems driven by dichotomous noise can often be described in full analytic detail. Second, dichotomous noise reduces to white shot noise and Gaussian white noise in the appropriate limits [2]. Third, it can either mimic the effects of finite correlation time of the noise, or it may directly provide a good representation of an actual physical situation (such as, for example, thermal transitions between two configurations or states). Finally, it has the advantage that it can easily be implemented as an external noise with finite support.

Most of the results for dynamics driven by dichotomous Markov noise are limited to systems with a single scalar variable $x(t)$. The rate of change of this variable switches at random between the " + " dynamics, $\dot{x}(t)=f_{+}(x)$, and the "- " dynamics, $\dot{x}(t)=f_{-}(x)$ (the dot stands for the temporal derivative). This dynamics can be described by the following stochastic differential equation:

$$
\dot{x}(t)=\frac{f_{+}(x)+f_{-}(x)}{2}+\frac{f_{+}(x)-f_{-}(x)}{2} \xi(t),
$$

where $\xi(t)$ is a realization of the dichotomous Markov process taking the values \pm 1 , with transition rates between these values equal to $k_{+}$and $k_{-}$, respectively. Several physical applications, including persistent random walks, nuclear magnetic resonance, chromatography, Taylor dispersion, and kinetic theory, have been discussed when the speeds $f_{+}$and $f_{-}$are constants, and exact time-dependent results can be obtained in this case [3]. Somewhat surprisingly, the full time-dependent solution for linear $f_{+}(x)$ and $f_{-}(x)$ is not available, see Ref. [4] for a detailed discussion. In the case of nonlinear dynamics, steady state properties can be calculated and have notably been studied in detail in the context of noise-induced transitions [5] and noise-induced phase transitions [6]. Specific dynamic properties have also been obtained. A significant effort has gone into the calculation of first passage time moments [7] and of transition rates, cf. Kramers' rate for thermal escape [8] and resonant activation [9]. Furthermore, when $f_{+}(x)$ and $f_{-}(x)$ are periodic, another important dynamic property (namely, the asymptotic drift velocity), can be extracted from steady state results by working with periodic boundary conditions. This technique is of particular interest in the context of Josephson junctions and Brownian motors [10-13]. However, contrary to the claims made in some of these papers, the problem of the first passage time moments, and the related issue of finding the asymptotic drift velocity [14], was not solved in the most general case. Indeed, with a few exceptions [15-18], all the results that have been obtained exclude the cases where one or both of the " \pm " dynamics have unstable fixed points, and thus do not consider the possibility of crossing unstable fixed points in the long time dynamics. The technical subtleties were first highlighted and discussed in detail in a recent paper [19]. In view of the broad applicability and importance of dichotomous Markov noise, a comprehensive review of the results for the asymptotic drift velocity in periodic systems driven by such a noise is called for and is the subject of this paper. As an illustration we also apply these results to the calculation of the drift for the rocking ratchet. 


\section{SOLVING THE MASTER EQUATION}

The calculation of the asymptotic drift velocity in periodic systems is most easily carried out by starting from the master equation, equivalent to Eq. (1), for the probability densities $P_{+}(x, t)$ and $P_{-}(x, t)$ to be at $x$ at time $t$, if $\xi=+1$ and -1 , respectively,

$$
\begin{aligned}
\frac{\partial P_{+}(x, t)}{\partial t}= & -\frac{\partial}{\partial x}\left[f_{+}(x) P_{+}(x, t)\right]-k_{+} P_{+}(x, t) \\
& +k_{-} P_{-}(x, t), \\
\frac{\partial P_{-}(x, t)}{\partial t}= & -\frac{\partial}{\partial x}\left[f_{-}(x) P_{-}(x, t)\right]-k_{-} P_{-}(x, t) \\
& +k_{+} P_{+}(x, t) .
\end{aligned}
$$

We assume that $f_{+}(x)$ and $f_{-}(x)$ are continuous functions of their argument, and that they are both periodic, i.e.,

$$
f_{+}(x)=f_{+}(x+L) \quad \text { and } \quad f_{-}(x)=f_{-}(x+L) \forall x .
$$

In order to extract the long-time average drift speed, it is sufficient to study the steady state properties of Eq. (2) for $x \in[0, L]$ with periodic boundary conditions. To show this, we introduce the steady state quantities $P(x)=P_{+}(x)$ $+P_{-}(x)$ (which represent the probability density for being at $x$ regardless of the value of $\xi)$ [27] and $p(x)=k_{+} P_{+}(x)$ $-k_{-} P_{-}(x)$. From the summation of the two equations in Eq. (2), one immediately concludes that for the asymptotic (steady) state the probability flux $J$ associated with $P(x)$,

$$
J=\frac{k_{+} f_{-}(x)+k_{-} f_{+}(x)}{k_{+}+k_{-}} P(x)+\frac{f_{+}(x)-f_{-}(x)}{k_{+}+k_{-}} p(x),
$$

is a constant. The asymptotic drift velocity is then simply given by

$$
\langle\dot{x}\rangle=\int_{0}^{L}\left[f_{+}(x) P_{+}(x)+f_{-}(x) P_{-}(x)\right] d x=L J .
$$

By subtracting the equations in Eq. (2) (multiplied, respectively, by $k_{+}$and $k_{-}$), one obtains, in the asymptotic steady state, the following first-order differential equation for $p(x)$ :

$$
\begin{array}{r}
\frac{d}{d x}\left\{k_{+} k_{-}\left[f_{+}(x)-f_{-}(x)\right] P(x)+\left[k_{+} f_{+}(x)\right.\right. \\
\left.\left.+k_{-} f_{-}(x)\right] p(x)\right\}+\left(k_{+}+k_{-}\right)^{2} p(x)=0 .
\end{array}
$$

Equations (4) and (6) have to be solved by imposing the conditions of continuity for $P(x)$ and $p(x)$ (or, at least, the condition that they have no more than integrable singularities) on $[0, L]$, periodicity $P(x)=P(x+L)$ and $p(x)=p(x$ $+L) \forall x$, and normalization for $P(x), \int_{0}^{L} P(x) d x=1$.

All these elements allow the determination of the unique steady state solution $P(x)$, the stationary probability flux $J$, and hence the corresponding dynamic quantity of interest to us, namely, the asymptotic drift velocity $\langle\dot{x}\rangle$. As was pointed out in detail in Ref. [19], the situation is entirely different, both physically and mathematically, when one or both of the " \pm " dynamics have unstable fixed points, depending on whether the system can cross (or not) these unstable fixed points in the long-time limit. In fact, while this issue was also mentioned in Refs. [15] and [16], a full and detailed discussion was first given for a specific example with multiplicative dichotomous noise in Ref. [19]. In the following, we focus on the presentation and discussion of the final results for the asymptotic probability density and drift velocity, relegating the technical details to the Appendix.

\section{MAIN RESULTS}

Since we assume that the functions $f_{ \pm}(x)$ are continuous, and in view of the periodicity, fixed points in the separate dynamics $\dot{x}=f_{+}(x)$ and $\dot{x}=f_{-}(x)$ will always appear in pairs. In the following, we will present the final results for the three simplest cases that can occur, namely, no fixed points, cf. Sec. III A, one of the dynamics has two fixed points and the other none, Sec. III B, and both dynamics have two fixed points, Sec. III C.

\section{A. No fixed points}

The stationary probability density $P(x)$ reads

$$
\begin{aligned}
P(x)= & \frac{\langle\dot{x}\rangle\left[f_{+}(x)-f_{-}(x)\right]}{L f_{+}(x) f_{-}(x)\left[\exp \left(\int_{0}^{L} d z\left[k_{+} / f_{+}(z)+k_{-} / f_{-}(z)\right]\right)-1\right]} \\
& \times \int_{x}^{x+L} d z\left[\frac{k_{+}+k_{-}}{f_{+}(z)-f_{-}(z)}+\left(\frac{f_{+}(z)+f_{-}(z)}{2\left[f_{+}(z)-f_{-}(z)\right]}\right)^{\prime}\right] \exp \left(-\int_{z}^{x} d w\left[k_{+} / f_{+}(w)+k_{-} / f_{-}(w)\right]\right),
\end{aligned}
$$

and the mean asymptotic velocity is given by 


$$
\begin{aligned}
\langle\dot{x}\rangle= & L\left[\exp \left(\int_{0}^{L} d z\left[k_{+} / f_{+}(z)+k_{-} / f_{-}(z)\right]\right)-1\right] \\
& \times\left\{\int _ { 0 } ^ { L } d x \frac { f _ { + } ( x ) - f _ { - } ( x ) } { f _ { + } ( x ) f _ { - } ( x ) } \int _ { x } ^ { x + L } d z \left[\frac{k_{+}+k_{-}}{f_{+}(z)-f_{-}(z)}\right.\right. \\
& \left.+\left(\frac{f_{+}(z)+f_{-}(z)}{2\left[f_{+}(z)-f_{-}(z)\right]}\right)^{\prime}\right] \exp \left(-\int_{z}^{x} d w\left[k_{+} / f_{+}(w)\right.\right. \\
& \left.\left.\left.+k_{-} / f_{-}(w)\right]\right)\right\}^{-1} .
\end{aligned}
$$

Here $(\cdots)^{\prime}$ stands for the derivative with respect to the argument (in the present case $z$ ). Note that the above expressions for both $P(x)$ and $\langle\dot{x}\rangle$ display the required symmetry between the "+" and "-" dynamics. The above expression reduces to the one given earlier in the literature for the particular case of additive dichotomous noise $\left[f_{+}(x)-f_{-}(x)\right.$ = const, cf. Ref. [11]].

\section{B. One of the dynamics has two fixed points in $[0, L)$}

We suppose that the "+" dynamics has two fixed points $x_{1}<x_{2}$ in $[0, L), x_{1}$ being stable $\left[f_{+}\left(x_{1}\right)=0, f_{+}^{\prime}\left(x_{1}\right)<0\right]$, while $x_{2}$ is unstable $\left[f_{+}\left(x_{2}\right)=0, f_{+}^{\prime}\left(x_{2}\right)>0\right]$. The "-" dynamics has no fixed points.

As explained in detail in part 2 of the Appendix and in Ref. [19], there exists exactly one solution $P(x)$ of the Eqs. (4) and (6), which is physically and mathematically acceptable, and for $x \in\left[x_{1}, x_{1}+L\right]$ it is given by the following expression:

$$
\begin{aligned}
P(x)= & \frac{\langle\dot{x}\rangle}{L}\left|\frac{f_{+}(x)-f_{-}(x)}{f_{+}(x) f_{-}(x)}\right| \int_{x_{2}}^{x} d z \operatorname{sgn}\left[\frac{f_{+}(z) f_{-}(z)}{f_{+}(z)-f_{-}(z)}\right] \\
& \times\left[\frac{k_{+}+k_{-}}{f_{+}(z)-f_{-}(z)}+\left(\frac{f_{+}(z)+f_{-}(z)}{2\left[f_{+}(z)-f_{-}(z)\right]}\right)^{\prime}\right] \\
& \times \exp \left(-\int_{z}^{x} d w\left[k_{+} / f_{+}(w)+k_{-} / f_{-}(w)\right]\right) .
\end{aligned}
$$

As discussed in part 2 of the Appendix, $P(x)$ is finite and continuous throughout the interval $\left(x_{1}, x_{1}+L\right)$, with, in particular, at the unstable fixed point $x_{2}$,

$$
\lim _{x \succ x_{2}} P(x)=\lim _{x \searrow x_{2}} P(x)=\frac{\langle\dot{x}\rangle}{L} \frac{\left(k_{+}+k_{-}\right) / f_{+}^{\prime}\left(x_{2}\right)+1}{f_{-}\left(x_{2}\right)\left[k_{+} / f_{+}^{\prime}\left(x_{2}\right)+1\right]}
$$

Note that positivity of $P(x)$ implies that $f_{-}(x)$ and $\langle\dot{x}\rangle$ must have the same sign. Hence the direction of the asymptotic drift is the same as that of the "-" dynamics, which is physically obvious.

At the stable fixed point $x_{1}, P(x)$ is either continuous when $k_{+} /\left|f_{+}^{\prime}\left(x_{1}\right)\right|>1$, i.e.,

$$
\lim _{x \searrow x_{1}} P(x)=\lim _{x \nearrow\left(x_{1}+L\right)} P(x)=\frac{\langle\dot{x}\rangle}{L} \frac{\left(k_{+}+k_{-}\right) /\left|f_{+}^{\prime}\left(x_{1}\right)\right|-1}{f_{-}\left(x_{1}\right)\left[k_{+} /\left|f_{+}^{\prime}\left(x_{1}\right)\right|-1\right]},
$$

or divergent but integrable when $k_{+} /\left|f_{+}^{\prime}\left(x_{1}\right)\right| \leqslant 1$ [namely, $k_{+} /\left|f_{+}^{\prime}\left(x_{1}\right)\right|<1$ corresponds to a power-law divergence, while $k_{+} /\left|f_{+}^{\prime}\left(x_{1}\right)\right|=1$ corresponds to a "marginal" logarithmiclike integrable divergence]. This result is consistent with the physical intuition that probability density builds up near a stable fixed point, especially when the switching rate is low.

The normalization condition $\int_{x_{1}}^{x_{1}+L} P(x) d x=1$, together with Eq. (5), leads to the following expression for the mean velocity:

$$
\begin{aligned}
\langle\dot{x}\rangle= & L\left\{\int_{x_{1}}^{x_{1}+L} d x\left|\frac{f_{+}(x)-f_{-}(x)}{f_{+}(x) f_{-}(x)}\right| \int_{x_{2}}^{x} d z \operatorname{sgn}\left[\frac{f_{+}(z) f_{-}(z)}{f_{+}(z)-f_{-}(z)}\right]\right. \\
& \times\left[\frac{k_{+}+k_{-}}{f_{+}(z)-f_{-}(z)}+\left(\frac{f_{+}(z)+f_{-}(z)}{2\left[f_{+}(z)-f_{-}(z)\right]}\right)^{\prime}\right] \\
& \left.\times \exp \left(-\int_{z}^{x} d w\left[k_{+} / f_{+}(w)+k_{-} / f_{-}(w)\right]\right)\right\}^{-1}
\end{aligned}
$$

This expression reduces to the one mentioned in Ref. [16] for the particular case of a symmetric additive dichotomous noise.

\section{Each of the alternating dynamics has two fixed points in $[0, L)$}

Consider as above $x_{1}<x_{2}$ as the stable, respectively unstable, fixed points of the "+" dynamics, and suppose now that the "-" dynamics also has two fixed points, $x_{3}<x_{4}$. Depending on the relative positions of these four points, as well as on their nature (stable or unstable), two different types of situations might occur.

(i) In all the situations in which $x_{1}$ has as direct neighbor another stable fixed point, it is physically clear that the asymptotic dynamics settles into a random alternating motion between these points, so that they delimit the interval in which the steady-state probability density is nonzero [5]. Obviously, in this case $\langle\dot{x}\rangle=0$, i.e., there is no net flux of the particles, while $P(x)$ is given by

$$
\begin{aligned}
P(x)= & C \frac{f_{+}(x)-f_{-}(x)}{f_{+}(x) f_{-}(x)} \\
& \times \exp \left(-\int_{x_{0}}^{x} d z\left[k_{+} / f_{+}(z)+k_{-} / f_{-}(z)\right]\right),
\end{aligned}
$$

for $x$ and $x_{0}$ lying between the two stable fixed points, while the constant $C$ is determined through the normalization condition for $P(x)$.

(ii) The cases that correspond to an alternation of the stable and unstable fixed points are of more interest to us, as they lead to a nontrivial behavior of $P(x)$ and to a nonzero 
flow of the particles. Without loss of generality we suppose that $0<x_{1}(s)<x_{3}(u)<x_{4}(s)<x_{2}(u)<L$, with the following results:

$$
P(x)=\left\{\begin{array}{l}
\frac{\langle\dot{x}\rangle}{L}\left|\frac{f_{+}(x)-f_{-}(x)}{f_{+}(x) f_{-}(x)}\right| \int_{x_{3}}^{x} d z \operatorname{sgn}\left[\frac{f_{+}(z) f_{-}(z)}{f_{+}(z)-f_{-}(z)}\right]\left[\frac{k_{+}+k_{-}}{f_{+}(z)-f_{-}(z)}+\left(\frac{f_{+}(z)+f_{-}(z)}{2\left[f_{+}(z)-f_{-}(z)\right]}\right)^{\prime}\right] \\
\quad \times \exp \left(-\int_{z}^{x} d w\left(k_{+} / f_{+}(w)+k_{-} / f_{-}(w)\right)\right) \text { for } x \in\left(x_{1}, x_{4}\right) \\
\frac{\langle\dot{x}\rangle}{L}\left|\frac{f_{+}(x)-f_{-}(x)}{f_{+}(x) f_{-}(x)}\right| \int_{x_{2}}^{x} d z \operatorname{sgn}\left[\frac{f_{+}(z) f_{-}(z)}{f_{+}(z)-f_{-}(z)}\right]\left[\frac{k_{+}+k_{-}}{f_{+}(z)-f_{-}(z)}+\left(\frac{f_{+}(z)+f_{-}(z)}{2\left[f_{+}(z)-f_{-}(z)\right]}\right)^{\prime}\right] \\
\quad \times \exp \left(-\int_{z}^{x} d w\left[k_{+} / f_{+}(w)+k_{-} / f_{-}(w)\right]\right) \text { for } x \in\left(x_{4}, x_{1}+L\right) .
\end{array}\right.
$$

$P(x)$ is continuous throughout $\left(x_{1}, x_{4}\right)$ and $\left(x_{4}, x_{1}+L\right)$ and, in particular, at the unstable fixed points $x_{2}$ and $x_{3}$ it takes, respectively, the values (10) and

$$
\lim _{x / x_{3}} P(x)=\lim _{x \backslash x_{3}} P(x)=\frac{\langle\dot{x}\rangle}{L} \frac{\left(k_{+}+k_{-}\right) / f_{-}^{\prime}\left(x_{3}\right)+1}{f_{+}\left(x_{3}\right)\left[k_{-} / f_{-}^{\prime}\left(x_{3}\right)+1\right]} .
$$

The periodicity of $P(x)$ is connected with the behavior at the stable fixed point $x_{1}$ that was already discussed in the preceding section, see Eq. (11).

Concerning the behavior of $P(x)$ at the other stable fixed point $x_{4}$, it is either divergent (but integrable) for $k_{-} /\left|f_{-}^{\prime}\left(x_{4}\right)\right| \leqslant 1$, or it is continuous,

$$
\lim _{x \backslash x_{4}} P(x)=\lim _{x \succ x_{4}} P(x)=\frac{\langle\dot{x}\rangle}{L} \frac{\left(k_{+}+k_{-}\right) /\left|f_{-}^{\prime}\left(x_{4}\right)\right|-1}{f_{+}\left(x_{4}\right)\left[k_{-} /\left|f_{-}^{\prime}\left(x_{4}\right)\right|-1\right]},
$$

when $k_{-} /\left|f_{-}^{\prime}\left(x_{4}\right)\right|>1$.

Imposing the normalization condition for $P(x)$, one obtains the corresponding asymptotic drift velocity:

$$
\begin{aligned}
\langle\dot{x}\rangle= & L\left\{\int_{x_{1}}^{x_{4}} d x\left|\frac{f_{+}(x)-f_{-}(x)}{f_{+}(x) f_{-}(x)}\right| \int_{x_{3}}^{x} d z \operatorname{sgn}\left[\frac{f_{+}(z) f_{-}(z)}{f_{+}(z)-f_{-}(z)}\right]\right. \\
& \times\left[\frac{k_{+}+k_{-}}{f_{+}(z)-f_{-}(z)}+\left(\frac{f_{+}(z)+f_{-}(z)}{2\left[f_{+}(z)-f_{-}(z)\right]}\right)^{\prime}\right] \\
& \times \exp \left(-\int_{z}^{x} d w\left[k_{+} / f_{+}(w)+k_{-} / f_{-}(w)\right]\right) \\
& +\int_{x_{4}}^{x_{1}+L} d x\left|\frac{f_{+}(x)-f_{-}(x)}{f_{+}(x) f_{-}(x)}\right| \int_{x_{2}}^{x} d z \operatorname{sgn}\left[\frac{f_{+}(z) f_{-}(z)}{f_{+}(z)-f_{-}(z)}\right] \\
& \times\left[\frac{k_{+}+k_{-}}{f_{+}(z)-f_{-}(z)}+\left(\frac{f_{+}(z)+f_{-}(z)}{2\left[f_{+}(z)-f_{-}(z)\right]}\right)^{\prime}\right] \\
& \left.\times \exp \left(-\int_{z}^{x} d w\left[k_{+} / f_{+}(w)+k_{-} / f_{-}(w)\right]\right)\right\}^{-1}, \quad(17)
\end{aligned}
$$

which completes the discussion.

All these results are suitable for a rapid generalization to cases when both dynamics have several pairs of fixed points. In particular, note that when there are several pairs of adjacent stable fixed points, the system is no longer ergodic. Depending on the initial conditions, the asymptotic motion of the particles is limited to one or another basin of attraction. These attractors are represented, on the real axis, by the intervals between such pairs of adjacent stable fixed points. We shall not go into further details here.

\section{ROCKING RATCHET}

The general formulas that were derived above still involve triple integrals. One interesting case for which explicit results can be obtained is that of piecewise constant functions $f_{+}$and $f_{-}$. To illustrate the results for this case, we will focus on one of the paradigms for Brownian motors, namely, the rocking ratchet [20]. In this problem an overdamped particle is gliding in a deterministic sawtooth-like potential $U(x)$ subject to an additional dichotomous forcing that

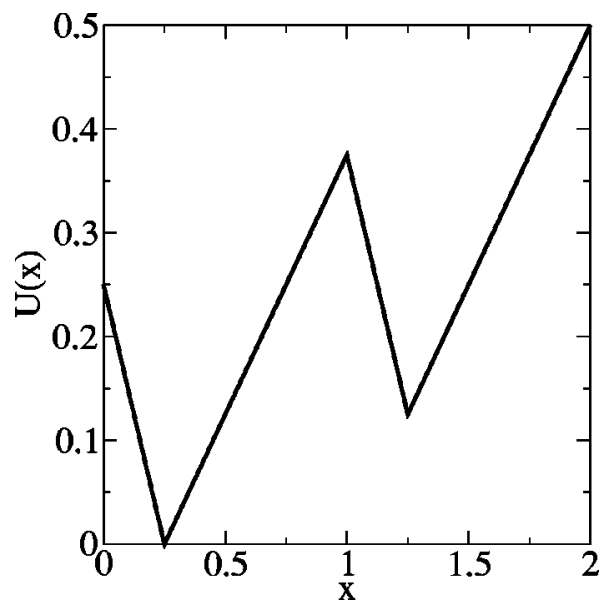

FIG. 1. The potential $U(x)$ used for numerical calculations on the rocking ratchet $\left(f_{1}=1.0, f_{2}=0.5, L=1.0\right.$, and $\left.L_{1}=0.25\right)$. 
switches between the values $A_{+}$and $-A_{-}$. The equation of motion is of the form

$$
\dot{x}=f(x)+\frac{A_{+}-A_{-}}{2}+\frac{A_{+}+A_{-}}{2} \xi(t),
$$

with $f(x)=-U^{\prime}(x)$, and thus $f_{ \pm}(x)=f(x) \pm A_{ \pm}$. Without loss of generality, we assume that the mean value of the forcing is zero, i.e., $A_{+} / k_{+}=A_{-} / k_{-}$, and, in order to fix ideas, we suppose that $A_{-} \geqslant A_{+}>0$ (the equality corresponds to a symmetric dichotomous noise). We now proceed to a presentation of the results as they follow from the more general discussion exposed earlier. Explicit closed analytical expressions for the stationary probability density and the mean asymptotic velocity can be obtained in the case of a continuous, piecewise linear force profile:

$$
f(x)= \begin{cases}f_{1} & \text { for } x \in\left[0, L_{1}-l\right) \\ f_{1}-\left(f_{1}+f_{2}\right)\left(x-L_{1}+l\right) / l & \text { for } x \in\left[L_{1}-l, L_{1}\right) \\ -f_{2} & \text { for } x \in\left[L_{1}, L_{1}+L_{2}-l\right) \\ -f_{2}+\left(f_{1}+f_{2}\right)\left(x-L_{1}-L_{2}+l\right) / l & \text { for } x \in\left[L_{1}+L_{2}-l, L_{1}+L_{2}\right),\end{cases}
$$

with $f(x+L)=f(x)$, where $L=L_{1}+L_{2}$; also, $f_{1}$ and $f_{2}$ are supposed to be two positive constants, with, e.g., $f_{2}<f_{1}$. However, the corresponding final formulas are rather lengthy and will not be presented here. Instead, we reproduce the results that are obtained by taking the (well-defined) limit $l \rightarrow 0$ in the final expressions for the asymptotic probability density and the mean velocity. Note that the limit $l \rightarrow 0$ corresponds, formally, to a periodic "block-wave" profile:

$$
f(x)=\left\{\begin{array}{l}
f_{1} \text { for } x \in\left[0, L_{1}\right) \\
-f_{2} \text { for } x \in\left[L_{1}, L_{1}+L_{2}\right) .
\end{array}\right.
$$

Although analytical calculations are done in full generality, all our numerical results were obtained for the potential represented in Fig. 1, i.e., we considered the case of a negative bias. In this case, the mean asymptotic velocity is determined both by a ratchetlike effect (if present) and by the bias of the potential. Of course, one can also consider (as usually done when studying ratchet effect) the case of an untilted potential, which corresponds to the condition $f_{1} L_{1}=f_{2} L_{2}$.

\section{A. Strong forcing: No fixed points}

When $A_{-}>f_{1}$ and $A_{+}>f_{2}$, running solutions appear for both tilts $+A_{+}$and $-A_{-}$, and there is no fixed point in any of the separate dynamics "+" and "-." Applying the results obtained in Sec. III A, one finds the following expression for the probability density in the two subintervals of one period $L$ :

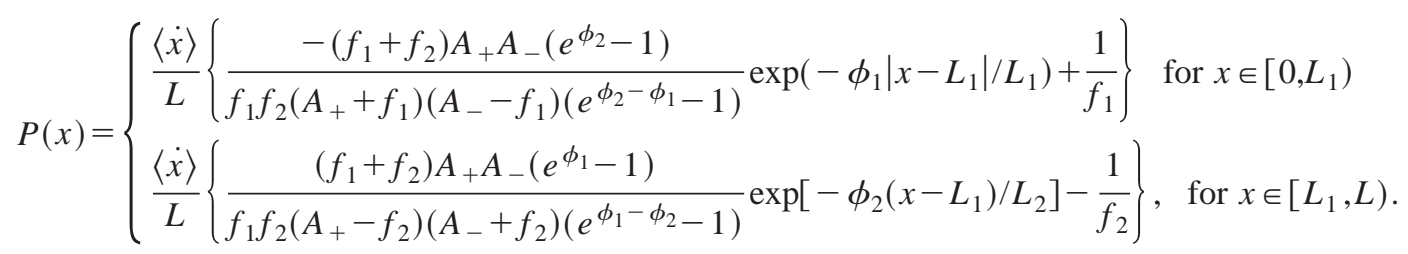

We used here the dimensionless quantities

$$
\phi_{1}=\frac{\left(k_{+}+k_{-}\right) f_{1} L_{1}}{\left(A_{-}-f_{1}\right)\left(A_{+}+f_{1}\right)} \text { and } \phi_{2}=\frac{\left(k_{+}+k_{-}\right) f_{2} L_{2}}{\left(A_{-}+f_{2}\right)\left(A_{+}-f_{2}\right)} .
$$

Because of the first-order discontinuity of $f(x)$ at $x=L_{1}, L, P(x)$ is also discontinuous at these points.

The mean asymptotic velocity reads

$$
\langle\dot{x}\rangle=L\left[\left(\frac{L_{1}}{f_{1}}-\frac{L_{2}}{f_{2}}\right)+\frac{A_{+} A_{-}}{k_{+}+k_{-}}\left(\frac{f_{1}+f_{2}}{f_{1} f_{2}}\right)^{2}\left(\frac{1-e^{-\phi_{1}-e^{\phi_{2}}+e^{\phi_{2}-\phi_{1}}}}{e^{\phi_{2}-\phi_{1}-1}}\right)\right]^{-1},
$$

and it is determined by the interplay between the characteristics of the noise and those of the potential (in particular, its bias, if any). Some limit cases of interest include the following.

(i) When $k_{ \pm} \rightarrow \infty$ (but $A_{ \pm}$finite) one finds, of course, $\langle\dot{x}\rangle=0$ - the noiseless (deterministic) result. 
(ii) The quenched noise limit $k_{ \pm} \rightarrow 0$ [i.e., a fraction $A_{-} /\left(A_{+}+A_{-}\right)$of the particles, chosen at random, are subjected to a constant external forcing $A_{+}$, while the remaining ones are subjected to an external forcing $\left.-A_{-}\right]$results in the following mean velocity:

$$
\langle\dot{x}\rangle=L \frac{f_{1} f_{2}\left(L_{1} f_{2}-L_{2} f_{1}\right)-A_{+} A_{-}\left(L_{1} f_{1}-L_{2} f_{2}\right)-L f_{1} f_{2}\left(A_{+}-A_{-}\right)}{\left(L_{1} f_{2}-L_{2} f_{1}\right)^{2}-L\left(A_{+}-A_{-}\right)\left(L_{1} f_{2}-L_{2} f_{1}\right)-L^{2} A_{+} A_{-}} .
$$

(iii) One obtains white shot noise [2] by taking the limit $A_{-} \rightarrow \infty, k_{-} \rightarrow \infty$, such that $A_{-} / k_{-}=A_{+} / k_{+} \equiv \lambda \neq 0 \quad\left(A_{+}\right.$ and $k_{+}$being finite). The mean velocity is then

$$
\begin{aligned}
\langle\dot{x}\rangle= & L\left[\left(\frac{L_{1}}{f_{1}}-\frac{L_{2}}{f_{2}}\right)+\lambda A_{+}\left(\frac{f_{1}+f_{2}}{f_{1} f_{2}}\right)^{2}\right. \\
& \left.\times \frac{1-e^{-\phi_{1}}-e^{\phi_{2}}+e^{\phi_{2}-\phi_{1}}}{e^{\phi_{2}-\phi_{1}}-1}\right]^{-1},
\end{aligned}
$$

where $\phi_{1}=f_{1} L_{1} /\left[\lambda\left(A_{+}+f_{1}\right)\right] \quad$ and $\quad \phi_{2}=f_{2} L_{2} /\left[\lambda\left(A_{+}\right.\right.$ $\left.\left.-f_{2}\right)\right]$. In the case of a symmetric potential $\left(f_{1}=f_{2}\right.$ and $L_{1}=L_{2}$ ), one recovers the result in [21].

Moreover, for very large values of $A_{+}$(and finite $\lambda$ ) one recovers the noiseless limit, namely, $\langle\dot{x}\rangle \approx\left(f_{1} L_{1}-f_{2} L_{2}\right) / L$ (that is strictly due to the bias of the potential).

(iv) Finally, the white noise limit: $A_{+}=A_{-} \equiv A \rightarrow \infty$ and $k_{+}=k_{-} \equiv k \rightarrow \infty$, with $A^{2} / 2 k=D$ finite. Then

$$
\phi_{1}=\frac{f_{1} L_{1}}{D} \quad \text { and } \quad \phi_{2}=\frac{f_{2} L_{2}}{D}
$$

and the mean velocity (23) takes the well-known form (see, e.g., Ref. [22]):

$$
\begin{aligned}
\langle\dot{x}\rangle= & L\left[\left(\frac{L_{1}}{f_{1}}-\frac{L_{2}}{f_{2}}\right)+D\left(\frac{f_{1}+f_{2}}{f_{1} f_{2}}\right)^{2}\right. \\
& \left.\times\left(\frac{1-e^{-\phi_{1}}-e^{\phi_{2}}+e^{\phi_{2}-\phi_{1}}}{e^{\phi_{2}-\phi_{1}}-1}\right)\right]^{-1} .
\end{aligned}
$$

In the case of an unbiased potential one has now $\phi_{1}=\phi_{2}$ $=\phi$, implying a zero mean velocity (as required by the second law of thermodynamics) and a probability density profile that assumes the Boltzmann form

$$
\begin{aligned}
P(x)= & \frac{f_{1} f_{2}}{\left(f_{1}+f_{2}\right) D\left(1-e^{-\phi}\right)} \\
& \times \begin{cases}\exp \left(-\phi\left|x-L_{1}\right| / L_{1}\right) & \text { for } x \in\left[0, L_{1}\right) \\
\exp \left[-\phi\left(x-L_{1}\right) / L_{2}\right] & \text { for } x \in\left[L_{1}, L\right) .\end{cases}
\end{aligned}
$$

\section{B. Intermediate forcing: Two fixed points}

For intermediate forcing, there are two situations that might occur.

(1) When $A_{-}>f_{1}$ but $A_{+}<f_{2}$, the "+" dynamics has two fixed points, namely, $x=L_{1}$ (stable, which corresponds to an asymmetric $\delta$ peak in the probability density), and $x$ $=L$ (unstable, which corresponds to a finite discontinuity in the probability density). One obtains from the results in Sec. III B:

$$
\begin{aligned}
P(x)= & -\frac{\langle\dot{x}\rangle}{L} \frac{2 A_{-}\left(A_{+}+f_{1}\right)\left(e^{\phi_{1}}-1\right)}{k_{+} f_{1}\left(A_{+}+A_{-}\right)} \delta_{-}\left(L_{1}-x\right)-\frac{\langle\dot{x}\rangle}{L} \frac{2 A_{-}\left(A_{+}-f_{2}\right)\left(e^{\phi_{2}-1}\right)}{k_{+} f_{2}\left(A_{+}+A_{-}\right)} \delta_{+}\left(x-L_{1}\right) \\
& - \begin{cases}\frac{\langle\dot{x}\rangle}{L f_{1}}\left[\frac{A_{-} e^{\phi_{1}}}{A_{-}-f_{1}} \exp \left[-\phi_{1}\left|x-L_{1}\right| / L_{1}\right]-1\right] & \text { for } \quad x \in\left[0, L_{1}\right) \\
\frac{\langle\dot{x}\rangle}{L f_{2}}\left[\frac{-A_{-} e^{\phi_{2}}}{A_{-}+f_{2}} \exp \left[-\phi_{2}\left(x-L_{1}\right) / L_{2}\right]+1\right] & \text { for } \quad x \in\left[L_{1}, L\right) .\end{cases}
\end{aligned}
$$

Here $\delta_{ \pm}(x)$ are the half Dirac- $\delta$ functions [28]. A simple intuitive explanation for their appearance is related to the fact that in the limit of the block-wave force $f(x)$, Eq. (20), a particle can reach the stable fixed point $x=L_{1}$ (coming either from its left or from its right) in a finite time, and, once there, it will stay at this position for the reminder of the "+" dynamics. Thus, in time average, this will give rise to a finite weight at this precise point. The corresponding mean velocity is then 


$$
\begin{aligned}
\langle\dot{x}\rangle= & -L\left\{\left(\frac{L_{2}}{f_{2}}-\frac{L_{1}}{f_{1}}\right)+\frac{A_{-}\left(A_{+}+f_{1}\right)\left(e^{\left.\phi_{1}-1\right)}\right.}{f_{1}}\left[\frac{1}{k_{+}\left(A_{+}+A_{-}\right)}+\frac{1}{\left(k_{+}+k_{-}\right) f_{1}}\right]\right. \\
& \left.+\frac{A_{-}\left(A_{+}-f_{2}\right)\left(e^{\left.\phi_{2}-1\right)}\right.}{f_{2}}\left[\frac{1}{k_{+}\left(A_{+}+A_{-}\right)}-\frac{1}{\left(k_{+}+k_{-}\right) f_{2}}\right]\right\}^{-1} .
\end{aligned}
$$

The direction of the mean velocity is, as expected, the same as that of the "-" dynamics, i.e., $\langle\dot{x}\rangle<0$ in this case.

One interesting limit in this case is that of white shot noise: $A_{-} \rightarrow \infty, k_{-} \rightarrow \infty$, so that $A_{-} / k_{-}=A_{+} / k_{+} \equiv \lambda$ finite. Our results are equivalent to those presented in Ref. [18] for the probability density (although written under a closed, compact form). One obtains the following expressions for the asymptotic velocity

$$
\langle\dot{x}\rangle=-L\left[\left(\frac{L_{2}}{f_{2}}-\frac{L_{1}}{f_{1}}\right)+\frac{\lambda\left(A_{+}+f_{1}\right)^{2}\left(e^{\phi_{1}}-1\right)}{A_{+} f_{1}^{2}}-\frac{\lambda\left(A_{+}-f_{2}\right)^{2}\left(e^{\phi_{2}}-1\right)}{A_{+} f_{2}^{2}}\right]^{-1} .
$$

Figure 2 shows the variation of the average asymptotic velocity as a function of $A_{+}$[according to Eqs. (25) and (31)] for various fixed values of the transition rate $k_{+}$. Note the peak and the discontinuity in the slope of the velocity at $A_{+}=f_{2}$, connected with the appearance of the fixed points when $A_{+}$decreases below $f_{2}$.

(2) When $A_{+}>f_{2}$ but $A_{-}<f_{1}$, there are no fixed points in the "+" dynamics, but two fixed points in the "- " dynamics, namely, $x=L_{1}$ (stable) and $x=L$ (unstable); together with the discontinuous character of $f(x)$ at these points, they lead, respectively, to an asymmetric $\delta$ peak of $P(x)$ at $x=L_{1}$ and to a first-order discontinuity of $P(x)$ at $x=L$ :

$$
\begin{aligned}
P(x)= & \frac{\langle\dot{x}\rangle}{L} \frac{2 A_{+}\left(A_{-}-f_{1}\right)\left(e^{\left.\phi_{1}-1\right)}\right.}{k_{-} f_{1}\left(A_{+}+A_{-}\right)} \delta_{-}\left(L_{1}-x\right)+\frac{\langle\dot{x}\rangle}{L} \frac{2 A_{+}\left(A_{-}+f_{2}\right)\left(e^{\left.\phi_{2}-1\right)}\right.}{k_{-} f_{2}\left(A_{+}+A_{-}\right)} \delta_{+}\left(x-L_{1}\right) \\
& + \begin{cases}\frac{\langle\dot{x}\rangle}{L f_{1}}\left[\frac{-A_{+} e^{\phi_{1}}}{A_{+}+f_{1}} \exp \left[-\phi_{1}\left|x-L_{1}\right| / L_{1}\right]+1\right] & \text { for } x \in\left[0, L_{1}\right) \\
\frac{\langle\dot{x}\rangle}{L f_{2}}\left[\frac{A_{+} e^{\phi_{2}}}{A_{+}-f_{2}} \exp \left[-\phi_{2}\left(x-L_{1}\right) / L_{2}\right]-1\right] & \text { for } x \in\left[L_{1}, L\right) .\end{cases}
\end{aligned}
$$

This leads to a mean velocity that reads

$$
\begin{aligned}
\langle\dot{x}\rangle= & L\left\{\left(\frac{L_{1}}{f_{1}}-\frac{L_{2}}{f_{2}}\right)+\frac{A_{+}\left(A_{-}-f_{1}\right)\left(e^{\phi_{1}}-1\right)}{f_{1}}\right. \\
& \times\left[\frac{1}{k_{-}\left(A_{+}+A_{-}\right)}-\frac{1}{\left(k_{+}+k_{-}\right) f_{1}}\right] \\
& +\frac{A_{+}\left(A_{-}+f_{2}\right)\left(e^{\phi_{2}}-1\right)}{f_{2}} \\
& \left.\times\left[\frac{1}{k_{-}\left(A_{+}+A_{-}\right)}+\frac{1}{\left(k_{+}+k_{-}\right) f_{2}}\right]\right\}^{-1} .
\end{aligned}
$$

Note that in this case $\langle\dot{x}\rangle>0$, as dictated by the sign of the "+" dynamics. One can thus clearly realize the role of the ratchet effect if, e.g., one considers a potential with negative bias (like the one in Fig. 1): indeed, in this case the mean velocity is directed against the bias. The above expression simplifies for the case of a symmetric dichotomic noise $A_{+}$ $=A_{-} \equiv A$ and $k_{+}=k_{-} \equiv k$, and reads

$$
\begin{aligned}
\langle\dot{x}\rangle= & L\left[\left(\frac{L_{1}}{f_{1}}-\frac{L_{2}}{f_{2}}\right)-\frac{\left(A-f_{1}\right)^{2}\left(e^{\phi_{1}}-1\right)}{2 k f_{1}^{2}}\right. \\
& \left.+\frac{\left(A+f_{2}\right)^{2}\left(e^{\phi_{2}}-1\right)}{2 k f_{2}^{2}}\right]^{-1} .
\end{aligned}
$$

\section{Weak forcing}

Finally, when $A_{-}<f_{1}$ and $A_{+}<f_{2}$, there are no freely running solution in either of the dynamics. The points $x$ $=L_{1}, L$ are fixed points for both "+" and "- " dynamics. One concludes that the mean asymptotic velocity is zero, and all the particles are concentrated at the stable fixed point $x$ $=L_{1}$, i.e., $P(x)=\delta\left(x-L_{1}\right)$. In this case additional thermal noise is needed to generate rectified motion. This problem has been solved for adiabatically slow forcing $[10,11]$.

\section{Comparison with numerical simulations}

The Langevin equation (18) is simulated numerically using 100000 particles and the probability distribution is aver- 


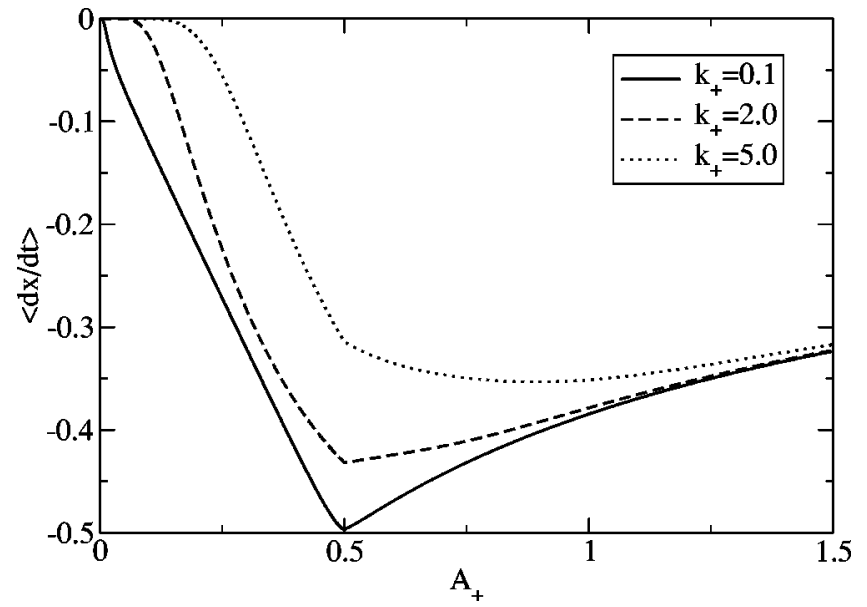

FIG. 2. For the white shot noise limit [Eqs. (25) and (31)]: The mean asymptotic velocity as a function of $A_{+}$for various values of the transition rate $k_{+}$. The sawtooth potential is the one represented in Fig. 1.

aged over ten snapshots. The biased potential shown in Fig. 1 is used for all numerical calculations. Figures 3 and 4 show, respectively, the probability distribution $P(x)$ and the average velocity $\langle\dot{x}\rangle$ for the strong forcing case, and Figs. 5 and 6 for the intermediate forcing case. Agreement between theory and simulations is very good. Discontinuities in $P(x)$ at $x=L_{1}$ and $x=L$ are clearly seen in both cases. Figure 5 also illustrates the existence of a $\delta$ peak at $x=L_{1}$. Note the phenomenon of current reversal when one decreases the amplitude of the perturbation (for a fixed $k$ ), i.e., when one passes from strong forcing (no fixed points, cf. Fig. 4) to intermediate forcing (fixed points appearing, cf. Fig. 6).

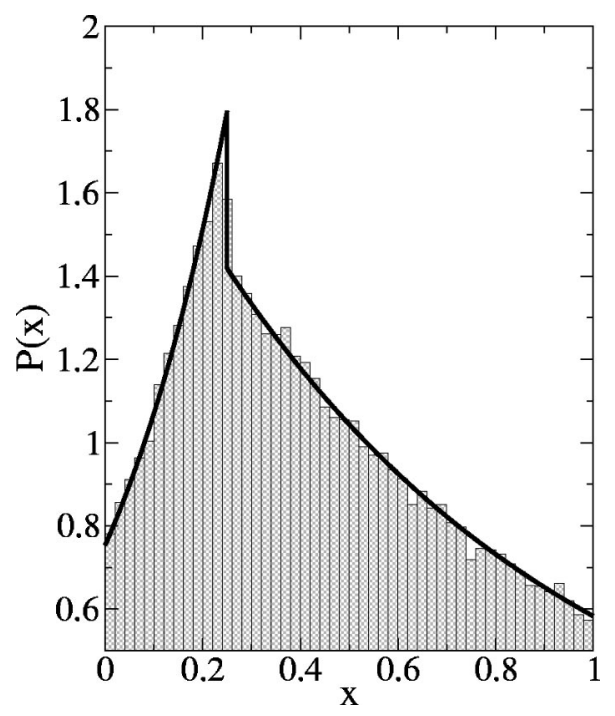

FIG. 3. Probability distribution $P(x)$ for the strong forcing case $\left[A_{+}=A_{-}=2.0\right.$ and $k_{+}=k_{-}=5.0$; see Fig. 1 for the parameters of $U(x)]$. The solid line shows the theoretical result (21) and the shaded histogram presents the results of numerical simulations.

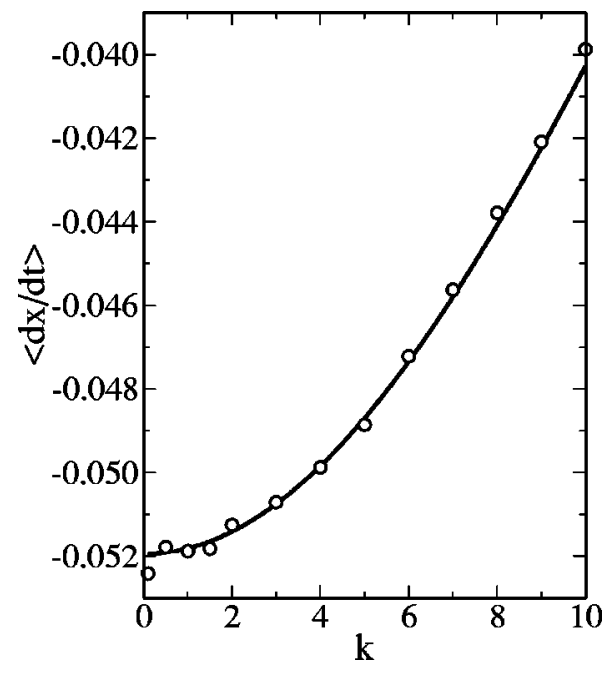

FIG. 4. Mean asymptotic velocity as a function of the transition rate $k$ for the strong forcing case (see Figs. 1 and 3 for parameter values). The solid line shows the theoretical result (23) and the open circles the results of numerical simulations.

\section{PERSPECTIVES}

The results obtained above reinforce the impression that dichotomous noise can be put on a par with Gaussian white noise as far as obtaining analytic results is concerned. Along this line of thought, we expect that one can obtain exact results for the first passage time moments when unstable fixed points are crossed. Also one can extend the calculations for the asymptotic diffusion coefficient in periodic systems subject to additive Gaussian white noise $[23,24]$ to the case of dichotomous forcing via its relationship to the first two moments of the first passage time [14]. Furthermore, the results presented here can be directly applied to various other problems, including Stokes' drift [13] and hypersensitive transport [25].

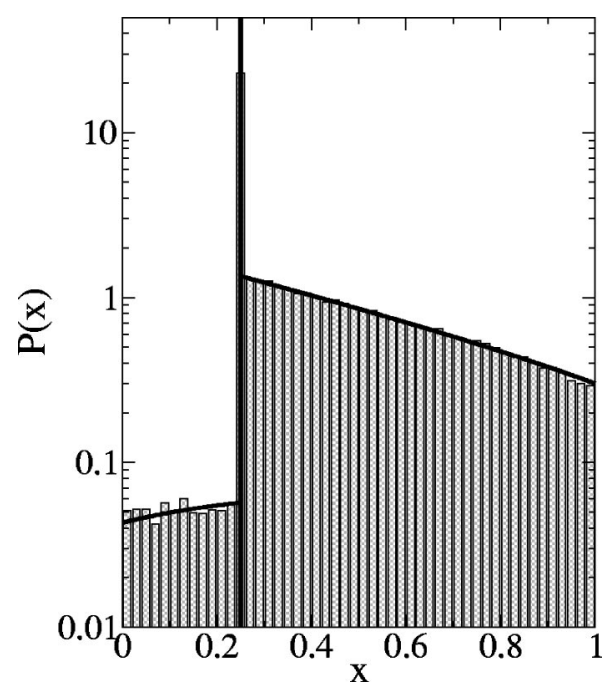

FIG. 5. Probability distribution $P(x)$ for the intermediate forcing case $\left[A_{+}=A_{-}=0.75, k_{+}=k_{-}=0.5\right.$; see Fig. 1 for the parameters of $U(x)]$. The solid line shows the theoretical result (29) and the shaded histogram presents the results of numerical simulations. 


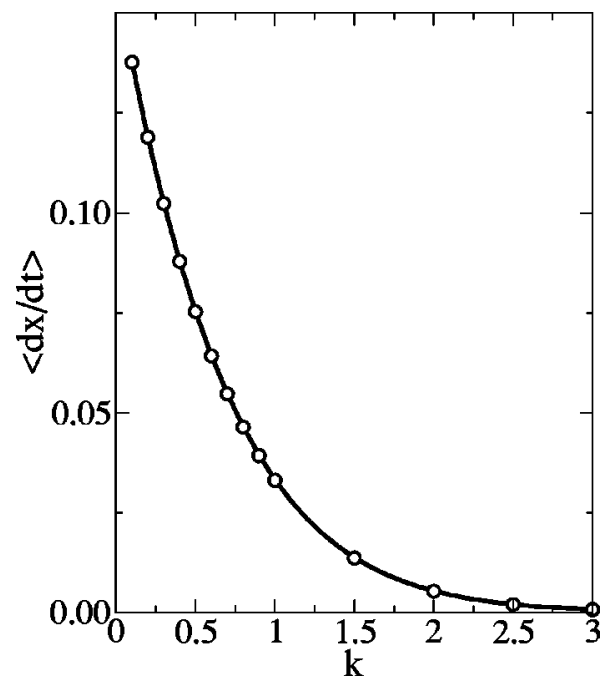

FIG. 6. Particle velocity as a function of the transition rate $k$ for the intermediate forcing case (see. Figs. 1 and 3 for parameter values). The solid line is the theoretical result (30) and the open circles result from numerical simulations.

\section{ACKNOWLEDGMENTS}

We acknowledge support from the Swiss National Science Foundation (I.B.), the National Science Foundation under Grant Nos. PHY-9970699 (K.L.) and DMS-0079478 (R.K.), the STOCHDYN program of the European Science Foundation (C.V.d.B.), and a grant from the New Del Amo Program. We also thank Professors P. Talkner and P. Hänggi for drawing our attention to Refs. [15,16,26].

\section{APPENDIX}

From the point of view of the mathematical steps involved in solving Eqs. (4) and (6), there are several cases that have to be considered separately:

Case I. When the coefficient of $p(x)$ in Eq. (4), namely $\left[f_{+}(x)-f_{-}(x)\right]$, has no zeros in $[0, L)$, one can solve this equation for $p(x)$.

Case II. When the coefficient of $P(x)$ in Eq. (4), i.e., $\left[k_{+} f_{-}(x)+k_{-} f_{+}(x)\right]$, has no zeros in $[0, L)$, one can solve Eq. (4) for $P(x)$. Note that cases I and II do not necessarily exclude one another.

Case III. When both the coefficients of $p(x)$ and of $P(x)$ in Eq. (4) have zeros, but not the same zeros, in $[0, L)$.

Case IV. When $f_{+}(x)$ and $f_{-}(x)$ have (at least) a common zero in $[0, L)$. In this case one can directly notice that there is no net flow of particles, i.e., $J=0$, given the existence of the common fixed point that cannot be crossed in any of the two dynamics. This case will hence not be studied any further.

A detailed analysis reveals that the results obtained in cases I and II are completely equivalent and apply also when $\left[f_{+}(x)-f_{-}(x)\right]$ and/or $\left[k_{+} f_{-}(x)+k_{-} f_{+}(x)\right]$ have different zeros in $[0, L]$, i.e., in case III. We therefore focus exclusively on the main results pertaining to case I.

In case I one can solve Eq. (4) with respect to $p(x)$, i.e.,

$$
p(x)=\frac{J\left(k_{+}+k_{-}\right)-\left[k_{+} f_{-}(x)+k_{-} f_{+}(x)\right] P(x)}{f_{+}(x)-f_{-}(x)},
$$

and by substitution in Eq. (6) one can obtain a closed firstorder differential equation for $P(x)$ :

$$
\begin{aligned}
& {\left[f_{+}(x)\right.}\left.f_{-}(x)\right] P^{\prime}(x)+\left\{\left[f_{+}(x) f_{-}(x)\right]^{\prime}-\left[f_{+}(x) f_{-}(x)\right]\right. \\
&\left.\times\left[\ln \left|f_{+}(x)-f_{-}(x)\right|\right]^{\prime}+\left[k_{+} f_{-}(x)+k_{-} f_{+}(x)\right]\right\} P(x) \\
&= J\left\{\left(k_{+}+k_{-}\right)+\left[f_{+}(x)-f_{-}(x)\right]\right. \\
&\left.\quad \times\left[\frac{f_{+}(x)+f_{-}(x)}{2\left[f_{+}(x)-f_{-}(x)\right]}\right]^{\prime}\right\} .
\end{aligned}
$$

Recall that $(\cdots)^{\prime}$ denotes derivation with respect to $x$.

The crux of the problem resides in finding the correct solution to Eq. (A2) [26]. A blind application of the standard method of variation of parameters leads to the familiar solution

$$
P(x)=\frac{\langle\dot{x}\rangle}{L}\left|\frac{f_{+}(x)-f_{-}(x)}{f_{+}(x) f_{-}(x)}\right|\left[C G\left(x, x_{0}\right)+K\left(x, x_{0} ; x\right)\right],
$$

where $C$ is a constant of integration that arises from the general solution to the homogeneous part of Eq. (A2), the second contribution is the particular solution of the full inhomogeneous equation, $x_{0}$ is an arbitrary point in $[0, L)$, and we have defined the functions

$$
\begin{gathered}
G(u, v)=\exp \left\{-\int_{v}^{u} d z\left[\frac{k_{+}}{f_{+}(z)}+\frac{k_{-}}{f_{-}(z)}\right]\right\} \\
K(u, v ; w)=\int_{v}^{u} d z \operatorname{sgn}\left[\frac{f_{+}(z) f_{-}(z)}{f_{+}(z)-f_{-}(z)}\right]\left[\frac{k_{+}+k_{-}}{f_{+}(z)-f_{-}(z)}\right. \\
\left.+\left(\frac{f_{+}(z)+f_{-}(z)}{2\left[f_{+}(z)-f_{-}(z)\right]}\right)^{\prime}\right] G(w, z) .
\end{gathered}
$$

The point is now to get the correct integration constant $C$, or rather constants, since one cannot apply the above solution at the points where the differential equation for $P(x)$ is singular, which is precisely at the location of fixed points, i.e., at the zeros of $f_{ \pm}$.

\section{No fixed points: $f_{+}(x) f_{-}(x) \neq 0$ in $[0, L)$}

In this case there is no fixed point in any of the alternating dynamics " +" and "-." The usual procedure to determine $C$ in Eq. (A3) can now be followed, i.e., we require periodicity of $P(x)$, i.e., $P(x)=P(x+L)$, recalling that both $f_{+}(x)$ and $f_{-}(x)$ are periodic. This results in Eq. (7) given in the main text. The mean velocity at the steady state, cf. Eq. (8) in the main text, follows from the normalization of $P(x)$.

\section{One of the alternating dynamics has two fixed points in $[0, L)$}

The situation is entirely different, both physically and mathematically, when the system can cross unstable fixed points within the interval $[0, L)$ in the long-time limit. Consider the simple case described in Sec. III B in the main text. 
Clearly, the steady-state results leading to Eqs. (4) and (6) still apply, but the solution of Eq. (6) is more delicate than the "blind" integration that led to expression (A3) for $P(x)$. Indeed, in the vicinity of the fixed points $x_{1}$ and $x_{2}$, if we simply try to apply formula (A3), we are led to the dependence

$$
P(x) \sim\left|x-x_{1,2}\right|^{-1-k_{+} /\left[f_{+}^{\prime}\left(x_{1,2}\right)\right]} .
$$

For the case of the stable fixed point, $P(x)$ is therefore continuous when $k_{+} /\left|f_{+}^{\prime}\left(x_{1}\right)\right|>1$, and divergent but integrable for $k_{+} /\left|f_{+}^{\prime}\left(x_{1}\right)\right| \leqslant 1$, a result that causes no conceptual difficulties. However, at the unstable fixed point $x_{2}$, this proce- dure leads to an apparent nonintegrable divergence, which is clearly unphysical, and mathematically improper in view of the requirement of normalization of $P(x)$.

As explained in detail in Ref. [19], the fallacy lies in the assumption that a single integration constant $C$, see Eq. (A3), is valid throughout the region $[0, L]$. One solves the problem by choosing different integration constants in each of the separate intervals $\left[0, x_{1}\right),\left(x_{1}, x_{2}\right)$, and $\left(x_{2}, L\right]$ between the fixed points. There is exactly one choice of this constant valid for both $\left(x_{1}, x_{2}\right)$ and $\left(x_{2}, L\right)$ such that the divergence at $x_{2}$ is removed, namely, $C=-K\left(x_{2}, x_{0} ; x_{0}\right)$; and another choice valid in the interval $\left[0, x_{1}\right)$ that ensures the required continuity and periodicity of $P(x)$. The acceptable expression for the probability density is therefore found to be

$$
P(x)= \begin{cases}\frac{\langle\dot{x}\rangle}{L}\left|\frac{f_{+}(x)-f_{-}(x)}{f_{+}(x) f_{-}(x)}\right|\left[K\left(L, x_{2} ; L\right) G(x, 0)+K\left(x, 0 ; x_{0}\right)\right] & \text { for } x \in\left[0, x_{1}\right) \\ \frac{\langle\dot{x}\rangle}{L}\left|\frac{f_{+}(x)-f_{-}(x)}{f_{+}(x) f_{-}(x)}\right| K\left(x, x_{2} ; x\right) & \text { for } x \in\left(x_{1}, L\right) .\end{cases}
$$

These expressions can be further simplified if one takes as the basic period not $[0, L]$, but $\left[x_{1}, x_{1}+L\right]$. Then the simplelooking, "compact" expression $P(x)=\langle\dot{x}\rangle / L \mid\left[f_{+}(x)\right.$ $\left.-f_{-}(x)\right] / f_{+}(x) f_{-}(x) \mid K\left(x, x_{2} ; x\right)$ [Eq. (9) in the main text] holds throughout this new basic period. With this choice of the basic interval, the normalization condition $\int_{x_{1}}^{x_{1}+L} P(x) d x=1$ leads to the expression (12) for the mean velocity.

$P(x)$ as given above [Eq. (A5) or Eq. (9)] meets all the requirements enumerated in Sec. II. In particular, let us check its behavior at the fixed points $x_{1}$ and $x_{2}$. In order to do this, it is useful to write Eq. (9) as

$$
P(x)=\frac{\langle\dot{x}\rangle}{L}\left|\frac{f_{+}(x)-f_{-}(x)}{f_{-}(x)}\right| \frac{K\left(x, x_{2} ; 0\right)}{\left|f_{+}(x)\right| G(0, x)} .
$$

For $x=x_{2}$ (the unstable fixed point), $K\left(x, x_{2} ; 0\right) /$ $\left\{\left|f_{+}(x)\right| G(0, x)\right\}$ presents an indeterminacy of the type " $0 / 0$;" applying Hôspital's rule one simply finds that $P(x)$ is continuous at $x=x_{2}$, and its value is given by Eq. (10).

For $x=x_{1}$ (the stable fixed point), there are three situations that might occur, depending on the value of $k_{+} /\left|f_{+}^{\prime}\left(x_{1}\right)\right|$ :

(a) For $k_{+} /\left|f_{+}^{\prime}\left(x_{1}\right)\right|>1$, both $K\left(x, x_{2} ; 0\right)$ and $\left|f_{+}(x)\right| G(0, x)$ present a divergence for $x \searrow x_{1}, x / x_{1}+L$; therefore, $P(x)$ presents an indeterminacy of the type " $\infty / \infty$." Applying Hôspital's rule one finds that $P(x)$ has the same finite limit as $x \searrow x_{1}, x / x_{1}+L$, as indicated in Eq. (11).

(b) For $k_{+} /\left|f_{+}^{\prime}\left(x_{1}\right)\right|<1, K\left(x, x_{2} ; 0\right)$ is finite and nonzero at $x=x_{1}$ and $x_{1}+L$, while $\left|f_{+}(x)\right| G(0, x) \rightarrow 0$

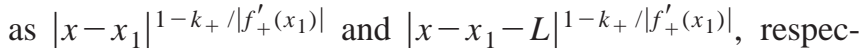

tively. Therefore, $P(x)$ presents a power-law integrable divergence, $P(x) \sim\left|x-x_{1}\right|^{-1+k_{+} /\left|f_{+}^{\prime}\left(x_{1}\right)\right|}$ in the neighborhood of $x_{1}$, and $P(x) \sim\left|x-x_{1}-L\right|^{-1+k_{+}}|| f_{+}^{\prime}\left(x_{1}\right) \mid$ in the neighborhood of $x_{1}+L$.

(c) Finally, for $k_{+} /\left|f_{+}^{\prime}\left(x_{1}\right)\right|=1, K\left(x, x_{2} ; 0\right)$ behaves like $\ln \left|x-x_{1}\right|$ in the vicinity of $x_{1}$ (respectively, like $\ln \left|x-x_{1}-L\right|$ in the vicinity of $\left.x_{1}+L\right)$, while $\left|f_{+}(x)\right| G(0, x)$ has a finite limit at these points. Thus, $P(x)$ has a "marginal," logarithmiclike divergence at these points.

\section{Each of the two alternating dynamics has two fixed points in $[0, L)$}

Suppose that each of the two dynamics, "+" and "-, ," has two fixed points (one stable and one unstable) in the interval $[0, L)$, i.e., the situation described in Sec. III B. Again, if one blindly applies the result (A3) for $P(x)$, then in the vicinity of the fixed points

$$
\begin{aligned}
& P\left(x-x_{1,2}\right) \sim\left|x-x_{1,2}\right|^{-1-k_{+} /\left[f_{+}^{\prime}\left(x_{1,2}\right)\right],} \\
& P\left(x-x_{3,4}\right) \sim\left|x-x_{3,4}\right|^{-1-k_{-} /\left[f_{-}^{\prime}\left(x_{3,4}\right)\right]} .
\end{aligned}
$$

One again encounters the nonphysical, nonintegrable divergence at the unstable fixed points. The correct procedure is again to use the solution (A3), but with different integration constants $C$ in each of the open intervals between the fixed points. The latter constants are determined by the requirements (i) to remove the strong divergences at the unstable fixed points, by imposing the condition that the coefficient of 
the divergent term becomes zero at these points; (ii) to ensure continuity and periodicity of $P(x)$; and (iii) through the normalization condition, to determine the flow $J$ and the mean asymptotic velocity $\langle\dot{x}\rangle$.

Case a: $0 \leqslant x_{1}(s)<x_{3}(u)<x_{4}(s)<x_{2}(u)<L$. The above "program" leads to the expression (14) in the main text for the probability density (in the appropriately chosen basic period $\left.\left[x_{1}, x_{1}+L\right]\right)$, and to the corresponding mean asymptotic velocity, Eq. (17).

Case b: $0 \leqslant x_{1}(s)<x_{2}(u)<x_{3}(s)<x_{4}(u)<L$. One gets the following expression for the probability density, again in the basic interval $\left[x_{1}, x_{1}+L\right]$ :

$$
P(x)= \begin{cases}\frac{\langle\dot{x}\rangle}{L}\left|\frac{f_{+}(x)-f_{-}(x)}{f_{+}(x) f_{-}(x)}\right| K\left(x, x_{2}, x\right) & \text { for } x \in\left(x_{1}, x_{3}\right) \\ \frac{\langle\dot{x}\rangle}{L}\left|\frac{f_{+}(x)-f_{-}(x)}{f_{+}(x) f_{-}(x)}\right| K\left(x, x_{4}, x\right) & \text { for } x \in\left(x_{3}, x_{1}+L\right),\end{cases}
$$

with the good behaviors (continuity) at the unstable fixed points $x_{2}$ and $x_{4}$ and either continuity or (integrable) divergences at the stable fixed points $x_{1}$ and $x_{3}$, as well as the required periodicity for $P(x)$. The mean asymptotic velocity is therefore given by

$$
\langle\dot{x}\rangle=L\left\{\int_{x_{1}}^{x_{3}} d x\left|\frac{f_{+}(x)-f_{-}(x)}{f_{+}(x) f_{-}(x)}\right| K\left(x, x_{2}, x\right)+\int_{x_{3}}^{x_{1}+L} d x\left|\frac{f_{+}(x)-f_{-}(x)}{f_{+}(x) f_{-}(x)}\right| K\left(x, x_{4}, x\right)\right\}^{-1} .
$$

[1] N.G. Van Kampen, Stochastic Processes in Physics and Chemistry (North-Holland, Amsterdam, 1992).

[2] C. Van den Broeck, J. Stat. Phys. 31, 467 (1983).

[3] R. Furth, Z. Phys. 2, 244 (1920); G.I. Taylor, Proc. London Math. Soc. 20, 196 (1921); S. Goldstein, Quart. J. Mech. Appl. Math. 4, 129 (1951); P.W. Anderson, J. Phys. Soc. Jpn. 9, 316 (1954); R. Kubo, ibid. 9, 935 (1954); J.C. Giddings and H. Eyring, J. Phys. Chem. 59, 416 (1955); W.C. Thacker, J. Phys. Oceanogr. 6, 66 (1959); H.P. McKean, J. Math. Phys. 8, 547 (1967); B. Gaveau, T. Jacobson, M. Kac, and L.S. Schulman, Phys. Rev. Lett. 53, 419 (1984).

[4] J.M. Sancho, J. Math. Phys. 25, 354 (1984); P. Jung, in Stochastic Dynamics, edited by L. Schimansky-Geier and T. Poschel (Berlin, Springer-Verlag, 1997), p. 23; V. Balakrishnan and C. Van den Broeck, Phys. Rev. E 65, 012101 (2001).

[5] W. Horsthemke and R. Lefever, Noise Induced Transitions (Springer-Verlag, Berlin, 1984).

[6] Seunghwan Kim, Seon Hee Park, and C.S. Ryu, Phys. Rev. E 58, 7994 (1998); C. Van den Broeck and R. Kawai, ibid. 57, 3866 (1998); S. Guillouzic and I. L'Heureux, ibid. 55, 5060 (1997).

[7] J.M. Sancho, Phys. Rev. A 31, 3523 (1985); J. Masoliver, K. Lindenberg, and B.J. West, ibid. 33, 2177 (1986); 34, 1481 (1986); 34, 2351 (1986); M.A. Rodriguez and L. Pesquera, ibid. 34, 4532 (1986); C.R. Doering, ibid. 35, 3166 (1987); V. Balakrishnan, C. Van den Broeck, and P. Hänggi, ibid. 38, 4213 (1988); U. Behn and K. Schiele, Z. Phys. B: Condens. Matter 77, 485 (1989); M. Kuś, E. Wajnryb, and K. Wódkiewicz, Phys. Rev. A 43, 4167 (1991); J. Olarrea, J.M.R. Parrondo, and F.J. de la Rubia, J. Stat. Phys. 79, 669 (1995); 79, 683 (1995).

[8] P. Reimann and T.C. Elston, Phys. Rev. Lett. 77, 5328 (1996).

[9] C.R. Doering and J.C. Gadoua, Phys. Rev. Lett. 69, 2318
(1992); C. Van den Broeck, Phys. Rev. E 47, 4579 (1993).

[10] M. Magnasco, Phys. Rev. Lett. 71, 1477 (1993); R.D. Astumian and M. Bier, ibid. 72, 1766 (1994).

[11] Ch.R. Doering, W. Horsthemke, and J. Riordan, Phys. Rev. Lett. 72, 2984 (1994); J. Kula, T. Czernik, and J. Luczka, Phys. Lett. A 214, 14 (1996); J. Luczka, T. Czernik, and P. Hanggi, Phys. Rev. E 56, 3968 (1997).

[12] V. Berdichevsky and M. Gitterman, Phys. Rev. E 56, 6340 (1997); M.M. Millonas and D.R. Chialvo, ibid. 53, 2239 (1996); S.H. Park, S. Kim, and C.S. Ryu, Phys. Lett. A 225, 245 (1997).

[13] I. Bena, M. Copelli, and C. Van den Broeck, J. Stat. Phys. 101, 415 (2000).

[14] D.R. Cox, Renewal Theory (Methuen \& Co., London, 1962); M. Khantha and V. Balakrishnan, Pramana 21, 111 (1983); C. Van den Broeck, in Noise and Nonlinear Phenomena in Nuclear Systems, edited by J.L. Munoz-Cobo and F.C. Difilippo (Plenum, 1989).

[15] U. Behn, R. Müller, and P. Talkner, Phys. Rev. E 47, 3970 (1993).

[16] I. Zapata, J. Luczka, F. Sols, and P. Hänggi, Phys. Rev. Lett. 80, 829 (1998).

[17] V. Balakrishnan, C. Van den Broeck, and I. Bena, Stochastics and Dynamics 1, 537 (2001).

[18] Results for the case of white shot noise, which can be obtained as a limit of the dichotomous Markov noise, were discussed in T. Czernik and J. Luczka, Ann. Phys. (Leipzig) 9, 721 (2000).

[19] I. Bena, C. Van den Broeck, R. Kawai, and Katja Lindenberg, Phys. Rev. E 66, 045603(R) (2002).

[20] P. Reimann, Phys. Rep. 361, 57 (2002) (for an overview and references therein); G. Carapella, Phys. Rev. B 63, 054515 (2001); E. Goldobin, A. Sterck, and D. Koelle, Phys. Rev. E 63, 031111 (2001); P. Reimann, in Lecture Notes in Physics, 
edited by J.A. Freund and T. Pöschel (Springer, Berlin, 2000), Vol. 557; E. Trias, J.J. Mazo, F. Falo, and T.P. Orlando, Phys. Rev. E 61, 2257 (2000); G.N. Milstein and M.V. Tretyakov, J. Phys. A 32, 5795 (1999); F. Falo, P.J. Martinez, J.J. Mazo, and S. Cilla, Europhys. Lett. 45, 700 (1999); C.-S. Lee, B. Janko, I. Derényi, and A.-L. Barabasi, Nature (London) 400, 337 (1999); J. Plata, Phys. Rev. E 57, 5154 (1998); M. Schreier, P. Reimann, P. Hänggi, and E. Pollak, Europhys. Lett. 44, 416 (1998); I. Derényi, C. Lee, and A.-L. Barabasi, Phys. Rev. Lett. 80, 1473 (1998); T.E. Dialynas, K. Lindenberg, and G.P. Tsironis, Phys. Rev. E 56, 3976 (1997); P. Hänggi and R. Bartussek, in Nonlinear Physics of Complex Systems, edited by J. Parisi, S.C. Müller, and W. Zimmermann, Lecture Notes in Physics Vol. 476 (Springer, Berlin, 1996); L. Gorre, E. Ioannidis, and P. Silberzan, Europhys. Lett. 33, 267 (1996); C.R. Doering, Nuovo Cimento D 17, 685 (1995); A. Ajdari, D. Mukamel, L. Peliti, and J. Prost, J. Phys. I 4, 1551 (1994); R. Bartussek, P. Hänggi, and J.G. Kissner, Europhys. Lett. 28, 459 (1994).

[21] J. Luczka, R. Bartussek, and P. Hänggi, Europhys. Lett. 31, 431 (1995).

[22] H. Risken, The Fokker-Planck Equation. Methods of Solution and Applications (Springer-Verlag, Berlin, 1984), Chap. 11.
[23] P. Reimann, C. Van den Broeck, H. Linke, P. Hänggi, M. Rubi, and A. Pérez-Madrid, Phys. Rev. Lett. 87, 010602 (2001); Phys. Rev. E 65, 031104 (2002).

[24] B. Lindner, M. Kostur, and L. Schimansky-Geier, Fluct. Noise Lett. 1, R25 (2001).

[25] M.B. Tarlie and R.D. Astumian, Proc. Natl. Acad. Sci. U.S.A. 95, 2039 (1998); V. Berdichevsky and M. Gitterman, Physica A 249, 88 (1998); S.L. Ginzburg and M.A. Pustovoit, Phys. Lett. A 291, 77 (2001); Phys. Rev. Lett. 80, 4840 (1998); J. Exp. Theor. Phys. 89, 801 (1999); O.V. Gerashchenko, S.L. Ginzburg, and M.A. Pustovoit, Eur. Phys. J. B 19, 101 (2001).

[26] I.N. Bronshtein and K.A. Semendyayev, in Handbook of Mathematics, 3rd ed., edited by K.A. Hirsch (Springer Verlag, New York, 1997).

[27] We shall assume that $P(x)$ is unique, i.e., that the system is ergodic. The necessary condition for this (that there are not several attractors of the dynamics) will be clarified subsequently.,

[28] This means $\quad \int_{-\infty}^{-0} \delta_{-}(x) f(x) d x=(1 / 2) \lim _{x / 0} f(x)$

$=(1 / 2) f(-0), \quad \int_{+0}^{\infty} \delta_{+}(x) f(x) d x=1 / 2 \lim _{x \backslash 0} f(x)$

$=1 / 2 f(+0)$, and $\delta(x)=\delta_{-}(x)+\delta_{+}(x)$. 